\title{
Utilização do custeio baseado em atividades em centro de material e esterilização como ferramenta gerencial*
}

\section{Using activities-based costing in a sterile processing department as a management tool}

\author{
Wellington Rodrigo de Souza ${ }^{1}$, Wilza Carla Spiri ${ }^{2}$, Silvana Andréa Molina Lima ${ }^{3}$,
} Andréa Bernardes ${ }^{4}$, Cláudia Helena Bronzatto Luppi ${ }^{5}$

\footnotetext{
* Texto extraído da dissertação de Utilização do custeio baseado em atividades em centro de material e esterilização como ferramenta gerencial.

${ }^{1}$ Enfermeiro, Mestre em Enfermagem. Enfermeiro do Hospital Estadual Bauru. Bauru, SP, Brasil. E-mail: wsouza.heb@famesp.org.br.

2 Enfermeira, Doutora em Enfermagem. Professora Assistente Doutora da Faculdade de Medicina de Botucatu da Universidade Estadual Paulista (FMB/UNESP) . Bauru, SP, Brasil. E-mail: wilza@fmb.unesp.br.

${ }^{3}$ Enfermeira, Doutorado em Ginecologia, Obstetrícia e Mastologia. Professora Assistente Doutora da FMB/UNESP. Bauru, SP, Brasil. E-mail: smolina@fmb.unesp.br.

${ }^{4}$ Enfermeira, Doutora em Enfermagem Fundamental. Professora Doutora da Escola de Enfermagem Ribeirão Preto da Universidade de São Paulo. Ribeirão Preto, SP, Brasil. E-mail: andreab@eerp.usp.br.

${ }^{5}$ Enfermeira, Doutorado em Bases Gerais da Cirurgia. Professora Assistente da FMB/UNESP. Bauru, SP, Brasil. E-mail: claudia@fmb.unesp.br.
}

\section{RESUMO}

O Centro de Materiais e Esterilização (CME) baseia-se nos custos que incidem nos processos desenvolvidos para seu gerenciamento e o custeio baseado em atividades $(A B C)$ pode ser uma ferramenta adequada para auxiliar o gestor nas decisões e prática gerencial. O objetivo do estudo foi avaliar a aplicação do custeio ABC para uma prática gerencial baseada em evidências e seu apoio ao processo de tomada de decisão. Estudo de caso, realizado em CME de hospital de ensino, usando análise documental para aplicação do custeio ABC. O custo individual da esterilização dos artigos foi de $R \$ 6,05$ (US\$ 3,23) e desinfecção foi de R\$ 3,03 (US\$ 1,61). O custeio ABC é aplicável e destaca evidências que balizam a tomada de decisão na prática gerencial, garantindo a análise do processo produtivo e intervenções decisivas e impactantes na gestão de custos.

Descritores: Custos e Análise de Custo; Controle de Custos; Administração de Serviços de Saúde; Administração de Materiais no Hospital.

\section{ABSTRACT}

The Center of Materials and Sterilization (CMS) is based on the cost related to the processes developed for its management and the activities-based costing $(A B C)$ can be an adequate tool to help the manager in management decisions and practice. The aim of the study was to evaluate the application of $A B C$ for an evidence-based management practice and its support for process of decision making. This is a case study, performed in a CMS of a teaching hospital, using documental analysis to apply the $A B C$. The individual cost to sterilize goods was $R \$ 6,05(U S \$ 3,23)$ and to disinfect was $R \$ 3,03$ (US\$ 1,61). The $A B C$ is applicable and highlights evidence supporting decision making in management practice, guaranteeing the analysis of the productivity process and the decisive and impacting interventions in the management of costs.

Descriptors: Costs and Cost Analysis; Cost Control; Health Services Administration; Materials Management, Hospital. 


\section{INTRODUÇÃO}

O gerenciamento de custos proporciona condições adequadas às instituições hospitalares para análise dos processos administrativos e assistenciais na busca do equilíbrio entre a oferta de serviços, disponibilidade de recursos financeiros, atendimento a critérios de qualidade e incorporação tecnológica.

A crescente demanda por serviços de saúde, promovida pelo princípio da universalização do acesso proposta pelo Sistema Único de Saúde (SUS), associada à adoção de novas opções tecnológicas levou a um descompasso entre os recursos financeiros disponíveis e os necessários para o atendimento dessa demanda ${ }^{(1-2)}$.

Essa equação, a princípio complexa, indica aos gestores das instituições hospitalares a importância da utilização de instrumentos gerenciais adequados à administração dos recursos utilizados na consecução de suas atividades, levando a uma crescente demanda pela gestão profissional $^{(2)}$.

Para tanto, conhecimento, desenvolvimento de habilidades e tomada de decisão baseada em evidências, da prática e do processo gerencial, são competências assertivas para esse aprimoramento e são capazes de promover ciclos de melhorias contínuas. Dessa forma, o gerenciamento de custos leva à eficácia e eficiência gerencial, sendo um instrumento fundamental para o controle de custos e por consequência para obtenção de resultados econômicos positivos em instituições públicas e privadas ${ }^{(3-4)}$.

Nesse cenário, destaca-se o gerenciamento de custos no Centro de Materiais e Esterilização (CME). Essa unidade é parte integrante do complexo hospitalar, inerente, indispensável e vital ao processo assistencial, sendo definido como unidade de apoio técnico, cuja finalidade é o fornecimento de artigos médicohospitalares adequadamente processados, proporcionando condições seguras para a assistência à saúde dos indivíduos enfermos e sadios ${ }^{(5-6)}$.

Considerada a importância do CME para a qualidade da assistência, torna-se imprescindível a reflexão sobre os custos que incidem sobre os processos desenvolvidos, bem como as análises econômicas, a fim de balizar o planejamento e a tomada de decisão por parte do enfermeiro gestor.

A identificação dos custos inerentes ao produto e as atividades do processo produtivo são os objetivos de um sistema de custeio, que tem por finalidade a geração de relatórios confiáveis, que subsidiem a decisão em diferentes unidades da instituição hospitalar ${ }^{(7)}$. Essas condições são contempladas no custeio baseado em atividades (custeio $A B C$ ), pois traz essa possibilidade aos gestores, proporcionando informações que permitem reestruturar as atividades identificadas para a melhoria da qualidade e eficiência ao processo, bem como otimização dos recursos financeiros.

$O$ custeio $A B C$ tem origem na sigla derivada do inglês "ABC" para "activity-based costing", sendo difundido no Brasil a partir da década de $90^{(8)}$, consistindo na alocação dos recursos nas diversas atividades que compõem o processo produtivo de um bem ou serviço, por meio dos objetos de custo. Identifica os recursos que foram utilizados nas atividades de produção de um determinado produto $^{(7,9)}$. A interdisciplinaridade na gestão de custos hospitalares reflete a necessidade de instrumentalização dos gestores de saúde na busca por capacitação e efetividade dos serviços de saúde $\mathrm{e}^{(1-2,4,10-12)}$.

$O$ enfermeiro tem utilizado o custeio $A B C$ na sua prática gerencial de maneira incipiente, não gerindo adequadamente os custos e dificultando a busca da eficiência assistencial. Contudo, há experiências que comprovam sua aplicabilidade no ambiente hospitalar, configurando-se em instrumento direcionador para a tomada de decisão na gestão de custos e também demonstram evidências sobre a acurácia do custeio $A B C$, entretanto, ainda não traduz-se em uma metodologia de uso efetivo no cotidiano de trabalho das organizações de saúde e no processo de trabalho da enfermagem ${ }^{(1,9,11,13)}$.

Sendo o custeio $A B C$ adequado ao ambiente hospitalar, com metodologia definida em literatura e como instrumento eficaz de gestão, tem-se então 
embasamento teórico para sua aplicação como ferramenta gerencial de apoio à tomada de decisão na gestão de custos em um CME, contribuindo para um processo gerencial baseado em evidências.

Portanto, este estudo pretendeu entender e empreender uma gestão baseada em evidências, por meio do custeio baseado em atividades para proporcionar informação e orientação apuradas, adequadas e precisas ao gerenciamento de enfermagem. Objetivou avaliar a aplicação do sistema de custeio baseado em atividades, no centro de material e esterilização de uma instituição hospitalar pública, visando uma prática gerencial baseada em evidências e seu consequente apoio ao processo de tomada de decisão.

\section{METODOLOGIA}

Pesquisa aplicada, descritiva, exploratória, tendo como procedimento técnico empregado o estudo de caso. Esse método tem por finalidade a observação e descrição dos aspectos de uma situação ou realidade bem como fornece informações valiosas e ricas em detalhes, baseadas em fontes de dados múltiplas e variadas, que permitem uma análise aprofundada de processos organizacionais e administrativos, sendo uma estratégia de pesquisa abrangente que convergiu ao objetivo dessa investigação ${ }^{(14-15)}$.

O campo de pesquisa foi o CME de um hospital público, estadual, de ensino, localizado no interior do Estado de São Paulo - Brasil. Destina-se exclusivamente a atendimento de usuários do SUS, sendo referência em prestação de serviços em saúde para 68 municípios nas diversas especialidades clínicas e cirúrgicas. Possui programa de residência médica, internato a estudantes de Medicina e campo de estágio para graduação de profissionais da saúde.

O estudo utilizou protocolo para realização de estudo de caso, constando de quatro etapas: planejamento, coleta e análise dos dados e conclusão. A etapa de coleta de dados, ocorreu entre fevereiro a maio de 2012 , sendo utilizadas a análise documental, a observação não participante e as atividades de Enfermagem validadas para $\mathrm{CME}^{(7)}$.

Para o cálculo de custos os valores foram expressos em Reais $(R \$)$ e foi considerado o valor de referência de US\$ 1,87/R\$ 1,00 (Taxa de câmbio em 31/12/2011). As fontes de coleta de dados foram os documentos disponibilizados pelo $\mathrm{CME}$, tais como: relatórios de custos, dos recursos humanos, consumo de insumos, entre outros. A técnica de observação foi realizada pelo pesquisador, de modo informal, a fim de complementar e/ou ratificar informações obtidas em outras fontes. As atividades inerentes ao CME foram elencadas a partir do estudo de validação dessas atividades ${ }^{(7)}$, comparando com aquelas contidas na análise documental.

Para a apresentação do caso foi considerada a ordenação dos dados feita segundo um modelo adaptado e adotado para a aplicação do custeio $A B C^{(7)}$, a saber:

- Passo 1: Diagnóstico institucional/unidade de análise;

- Passo 2: Mapeamento dos processos;

- Passo 3: Identificação das atividades;

- Passo 4: Mensuração dos recursos e custeio das atividades;

- Passo 5: Custeio dos objetos de custo.

Durante a realização do estudo foram obedecidas as recomendações da legislação brasileira para sua condução e o projeto foi aprovado pelo Comitê de Ética em Pesquisa da Universidade Estadual Paulista UNESP/Botucatu sob protocolo CEP 3954-2011.

\section{RESULTADOS}

\section{Aplicação do Custeio ABC}

\section{Passo 1 - Diagnóstico institucional/unidade de análise}

O hospital em estudo possui complexidade terciária e capacidade para internação em 318 leitos operacionais (internação clínica e cirúrgica adulta, pediátrica, 
queimaduras e terapia intensiva), 14 salas cirúrgicas, 30 salas/consultórios para atendimento ambulatorial.

O CME dessa instituição foi a unidade de análise deste estudo de caso. Localiza-se no piso inferior do prédio, constituído da área de expurgo, preparo, desinfecção, esterilização, armazenamento e distribuição, totalizando $326 \mathrm{~m}^{2}$. Lavadora ultrassônica, duas lavadoras/termo desinfetadoras, quatro autoclaves, uma autoclave de bancada e uma secadora de artigos médico-hospitalares respiratórios fazem parte do parque tecnológico do $\mathrm{CME}$, e assim, utiliza o vapor saturado sob pressão como único método de esterilização e o calor como método de desinfecção de artigos médico-hospitalares (desinfecção térmica).

A unidade está subordinada hierarquicamente à Gerência de Enfermagem das unidades assistenciais e possui 43 funcionários assim divididos: cinco enfermeiras, sendo uma a supervisora da unidade; 36 técnicos de Enfermagem e dois oficiais administrativos.

No ano de 2011, foram processados, mais de $496 \mathrm{mil}$ artigos médico-hospitalares. Os dados e informações gerados pelo processo foram registrados pela equipe de enfermagem e pelos oficiais administrativos sendo digitados em planilhas eletrônicas para posterior encaminhamento à assessoria financeira da instituição, que tem por finalidade a compilação das informações e o rateio do custo do CME para todos os clientes intrahospitalares.

\section{Passo 2 - Mapeamento dos processos}

Os artigos médico-hospitalares e insumos foram processados conforme demonstrado na Figura 1 e podese verificar a sequência das atividades em cada subprocesso.

\section{Passo 3 - Identificação das atividades}

A partir do delineamento do processo foram identificados os subprocessos e as atividades principais (Quadro 1).

\section{Passo 4 - Mensuração dos recursos e custeio das} atividades

Os recursos financeiros utilizados para realização das atividades no CME foram identificados por meio de relatório financeiro mensal, disponível na unidade.

Os dados foram organizados e compõem a Tabela 1.

Os valores constantes do relatório foram alocados nas 10 categorias constantes da Tabela 1 , descritas como recursos necessários às atividades do processo. Para cada recurso identificado dividiu-se o valor total por um denominador (direcionador), por exemplo, o gasto com pessoal foi dividido pelo tempo (horas) utilizado pelo funcionário em cada atividade realizada.

Assim, todos os recursos foram distribuídos entre todas as atividades, segundo os direcionadores de cada atividade constante do processo, conforme mostra a Tabela 2.

\section{Passo 5 - Custeio dos objetos de custo}

O CME tem como objetos de custo: número de artigos submetidos ao processo de esterilização e desinfecção térmica e o número de artigos processados por tipo de material (material leve/avulso, caixa/roupa pequena, caixa/roupa média, caixa grande).

Os critérios para classificação dos materiais foram a especificidade de uso para cada tipo de artigo e o tempo e temperatura da esterilização e desinfecção necessários para seu processamento. Assim, ajustou-se multiplicadores (pesos) para cada objeto de custo, a saber:

- Material leve/avulso - peso 1;

- $\quad$ Caixa/roupa pequena - peso 2

- Caixa/roupa média - peso 3;

- $\quad$ Caixa grande - peso 4;

Essa forma de classificação dos objetos de custo foi específica para o CME em estudo, podendo o gestor da unidade, adotar diferentes formas de classificação dos objetos de custo, conforme sua finalidade e perfil dos clientes atendidos. 


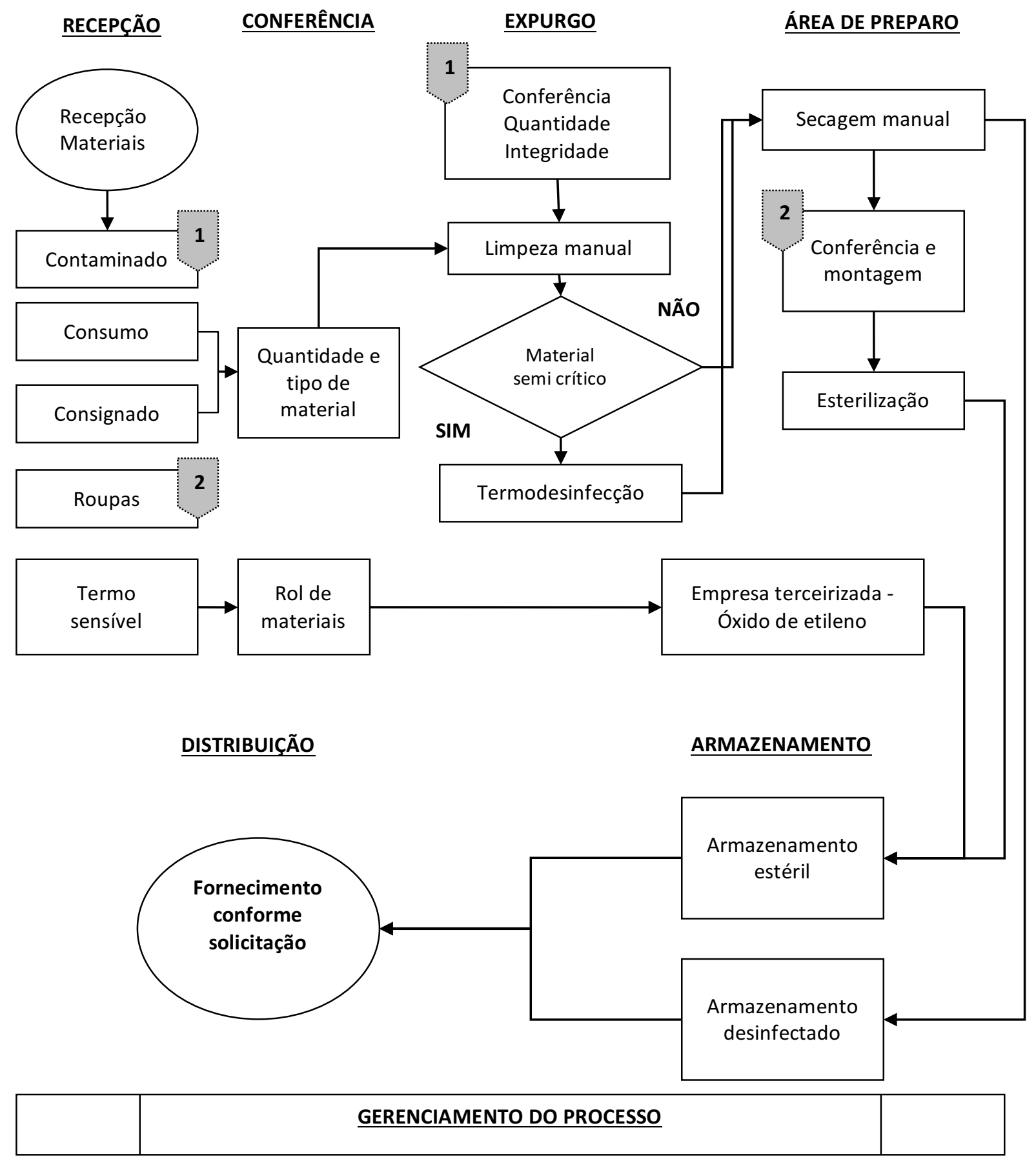

Figura 1: Fluxograma do processo do CME. Bauru, SP, Brasil, 2012. 
Quadro 1: Relação dos subprocessos e atividades. Bauru, SP, Brasil, 2012.

\begin{tabular}{|c|c|c|}
\hline Subprocessos & Código & Atividades \\
\hline \multirow{4}{*}{ RECEPÇÃO } & A1 & Recebimento de artigos médico-hospitalares \\
\hline & A2 & Recolhimento dos artigos médico-hospitalares \\
\hline & A3 & Conferência e registro dos artigos médico-hospitalares \\
\hline & A4 & Segregação dos artigos médico-hospitalares \\
\hline \multirow{4}{*}{ LIMPEZA } & B1 & Preparação de solução e montagem de rack/cesto \\
\hline & B2 & Imersão dos artigos médico-hospitalares em solução \\
\hline & B3 & Limpeza manual dos artigos médico-hospitalares \\
\hline & B4 & Colocação rack/cesto no equipamento \\
\hline DESINFECÇÃO & C1 & Desinfecção térmica \\
\hline \multirow{5}{*}{ PREPARO } & D1 & Secagem dos artigos médico-hospitalares \\
\hline & D2 & Conferência dos artigos médico-hospitalares \\
\hline & D3 & Montagem e rotulação dos artigos médico-hospitalares \\
\hline & D4 & Recebimento, conferência e montagem roupas \\
\hline & D5 & Encaminhamento de artigos médico-hospitalares (Terceirizado) \\
\hline \multirow{4}{*}{ ESTERILIZAÇÃO } & E1 & Montagem da carga \\
\hline & E2 & Acompanhamento dos ciclos de esterilização \\
\hline & E3 & Retirada da carga \\
\hline & E4 & Verificação da efetividade da esterilização \\
\hline \multirow{3}{*}{ ARMAZENAMENTO } & F1 & Acondicionamento e guarda dos artigos médico-hospitalares/roupas \\
\hline & $\mathrm{F} 2$ & Controle de validade dos artigos médico-hospitalares \\
\hline & F3 & Controle de temperatura e umidade da área \\
\hline \multirow{3}{*}{ DISTRIBUIÇÃO } & G1 & Montagem e distribuição de kit cirúrgico \\
\hline & G2 & Montagem de caixas com artigos médico-hospitalares \\
\hline & G3 & Distribuição de artigos médico-hospitalares \\
\hline GESTÃO & $\mathrm{H} 1$ & Gerenciamento do processo \\
\hline
\end{tabular}

Tabela 1: Matriz de recursos identificados, direcionadores e valores no CME, no ano de 2011. Bauru, SP, Brasil, 2012.

\begin{tabular}{cccc}
\hline Recurso & Direcionador & Valor R\$ & $\mathbf{\%}$ \\
\hline Pessoal & Tempo (horas) & $1.178 .175,72$ & $44,54 \%$ \\
Materiais de consumo e embalagens & \% de consumo & $471.872,80$ & $17,84 \%$ \\
Manutenção Equipamentos & Ordem de serviço & $331.601,01$ & $12,53 \%$ \\
Água & \% de consumo & $245.452,97$ & $9,28 \%$ \\
Materiais médico-odonto CME & Consumo direto (item) & $139.595,05$ & $5,28 \%$ \\
Lavanderia & Consumo direto (item) & $130.722,92$ & $4,94 \%$ \\
Manutenção predial & Ordem de serviço & $80.613,48$ & $3,05 \%$ \\
Energia Elétrica & Metragem - m ${ }^{2}$ & $29.394,44$ & $1,11 \%$ \\
Serviço de Higiene e Limpeza & Metragem - m ${ }^{2}$ & $34.654,07$ & $1,31 \%$ \\
Telefone & Número de ramais & $3.330,38$ & $0,13 \%$ \\
\hline
\end{tabular}

Fonte: Relatório financeiro individualizado por centro de custos. HEB, 2012 
Tabela 2: Matriz dos recursos por atividade. Bauru, SP, Brasil, 2012.

\begin{tabular}{|c|c|c|c|c|c|c|c|c|c|c|c|}
\hline Ativ. & Pessoal & Mat. Cons. Emb. & Man. Equip. & Água & Lavan-deria & Mat. CME & Energia Elétrica & SHL & Tele- fone & Man. Predial & TOTAL \\
\hline A1 & $7.001,28$ & $4.718,73$ & $15.423,30$ & - & - & & 99,18 & 116,93 & 832,60 & $2 b 72,01$ & $12.208,13$ \\
\hline $\mathrm{A} 2$ & $3.590,40$ & $4.718,73$ & & - & - & & 99,18 & 116,93 & - & 272,01 & $9.629,85$ \\
\hline A3 & $13.647,12$ & $4.718,73$ & $2.570,55$ & - & - & & 99,18 & 116,93 & - & 272,01 & $18.853,97$ \\
\hline A4 & $4.936,80$ & $4.718,73$ & & - & -- & & 99,18 & 116,93 & - & 272,01 & $10.143,65$ \\
\hline B1 & $2.513,28$ & $4.718,73$ & $15.423,30$ & $2.454,53$ & - & & 99,18 & 116,93 & - & 272,01 & $25.597,96$ \\
\hline B2 & $7.749,28$ & $4.718,73$ & $5.141,10$ & - & - & & 99,18 & 116,93 & - & 272,01 & $12.956,13$ \\
\hline B3 & $28.442,64$ & $4.718,73$ & $51.411,01$ & $4.909,06$ & & & 99,18 & 116,93 & - & 272,01 & $41.129,10$ \\
\hline B4 & $2.872,32$ & - & $11.995,90$ & - & - & & 99,18 & 116,93 & - & 272,01 & $3.360,44$ \\
\hline $\mathrm{C} 1$ & $14.600,96$ & - & & $41.727,00$ & - & & 108,20 & 127,57 & - & 296,74 & $72.283,77$ \\
\hline D1 & $364.515,36$ & $23.593,64$ & & - & $6.536,15$ & & $2.407,46$ & $2.838,23$ & - & $6.602,39$ & $411.634,33$ \\
\hline D2 & $27.376,80$ & $23.593,64$ & & - & $13.072,29$ & & $2.407,46$ & $2.838,23$ & - & $6.602,39$ & $128.134,42$ \\
\hline D3 & $475.428,80$ & $377.498,24$ & $44.984,63$ & - & $45.753,02$ & $67.165,73$ & $2.407,46$ & $2.838,23$ & 832,58 & $6.602,39$ & $989.689,78$ \\
\hline D4 & $34.264,56$ & $47.187,28$ & & - & $58.825,31$ & $67.165,73$ & $2.407,46$ & $2.838,23$ & - & $6.602,39$ & $219.290,97$ \\
\hline D5 & $5.697,56$ & $2.359,36$ & $8.568,50$ & - & - & & $2.407,46$ & $2.838,23$ & - & $6.602,39$ & $19.905,01$ \\
\hline E1 & $5.535,20$ & $4.718,73$ & $5.141,10$ & - & - & 2631,79 & $2.407,46$ & $2.838,23$ & - & $6.602,39$ & $24.733,80$ \\
\hline E2 & $14.897,52$ & - & $5.141,10$ & $196.362,38$ & - & & $2.407,46$ & $2.838,23$ & - & $6.602,39$ & $268.092,61$ \\
\hline E3 & $3.171,52$ & $4.718,73$ & & - & - & & $2.407,46$ & $2.838,23$ & - & $6.602,39$ & $19.738,33$ \\
\hline E4 & $4.158,88$ & $4.718,73$ & & - & $6.536,15$ & 2631,79 & $2.407,46$ & $2.838,23$ & - & $6.602,39$ & $38.462,13$ \\
\hline $\mathrm{F} 1$ & $12.985,28$ & $4.718,73$ & & - & - & & 736,36 & 871,67 & - & $2.019,46$ & $26.472,60$ \\
\hline $\mathrm{F} 2$ & $10.142,88$ & $4.718,73$ & & - & - & & 736,36 & 871,67 & - & $2.019,46$ & $23.630,20$ \\
\hline F3 & 89,76 & $4.718,73$ & $165.800,51$ & - & - & & 736,36 & 871,67 & - & $2.019,46$ & $8.435,98$ \\
\hline G1 & $18.694,94$ & $4.718,73$ & $15.423,30$ & - & - & & 736,36 & 871,67 & 832,60 & $2.019,46$ & $27.041,16$ \\
\hline $\mathrm{G} 2$ & $1.795,20$ & $4.718,73$ & & - & - & & 736,36 & 871,67 & - & $2.019,46$ & $10.141,42$ \\
\hline G3 & $10.069,84$ & $4.718,73$ & $2.570,55$ & - & - & & 736,36 & 871,67 & - & $2.019,46$ & $19.248,65$ \\
\hline $\mathrm{H} 1$ & $11.425,26$ & $14.580,87$ & & - & - & & $2.407,46$ & $2.838,23$ & 832,60 & $6.602,39$ & $204.487,31$ \\
\hline Total & $1.178 .175,72$ & $564.312,70$ & $331.601,01$ & $245.452,97$ & $130.722,92$ & $139.595,05$ & $29.394,44$ & $34.654,07$ & $3.330,38$ & $80.613,48$ & $2.645 .412,84$ \\
\hline
\end{tabular}




\section{Direcionadores das atividades}

A produção dos objetos de custo é feita por sucessivas atividades desenvolvidas em cada subprocesso. Assim, existe uma relação direta das diferentes atividades com os objetos de custo. Os valores dos objetos de custo foram calculados, somando o valor de todas as atividades empregadas no processamento dos objetos de custo pela quantidade de objetos de custo processados. Por exemplo, o objeto de custo "Desinfecção" não é contemplado com o subprocesso Esterilização, então verifica-se na Tabela 3 que essas atividades não fizeram parte do valor total da "Desinfecção". Por outro lado, vemos que todas as atividades são inerentes ao objeto de custo "Esterilização".

Quando se separam os artigos submetidos ao processo de esterilização nos diferentes objetos de custo usados pela unidade, têm-se outros valores de custo unitário dos artigos, devido à diferença no tempo de processamento dos mesmos ser justificada por suas dimensões e pesos (Tabela 3). Destaca-se que o valor do objeto de custo caixa/roupa pequena (R\$ 6,34) e caixa/roupa média $(\mathrm{R} \$ 6,18)$ têm valores relativamente semelhantes. O objeto de custo caixa grande atingiu o custo de $R \$ 14,16$, sendo o maior valor unitário de todos os objetos de custo.

Tabela 3: Custo dos objetos de custo por tipo de material: Desinfecção e Esterilização. Material leve/avulso e caixa/roupa pequena, média e grande. Bauru-SP, 2012

\begin{tabular}{|c|c|c|c|c|c|c|}
\hline Ativ. & Desinfecção & Esterilização & $\begin{array}{l}\text { Leve/avulso } \\
\text { (P1) }\end{array}$ & $\begin{array}{c}\text { Caixa/roupa } \\
\text { pequena (P2) }\end{array}$ & $\begin{array}{l}\text { Caixa/roupa } \\
\text { média (P3) }\end{array}$ & $\begin{array}{l}\text { Caixa/roupa } \\
\text { grande (P4) }\end{array}$ \\
\hline A1 & $4.347,22$ & $5.508,10$ & $2.927,22$ & $1.072,74$ & $1.241,20$ & 266,94 \\
\hline $\mathrm{A} 2$ & $4.347,22$ & $5.508,10$ & $2.927,22$ & $1.072,74$ & $1.241,20$ & 266,94 \\
\hline A3 & $8.694,44$ & $11.016,20$ & $5.854,44$ & $2.145,48$ & $2.482,40$ & 533,88 \\
\hline A4 & $4.347,22$ & $5.508,10$ & $2.927,22$ & $1.072,74$ & $1.241,20$ & 266,94 \\
\hline B1 & $25.614,61$ & $2.5614,61$ & $2.561,46$ & $5.122,92$ & $7.684,38$ & $10.245,84$ \\
\hline B2 & $13.293,49$ & $13.293,49$ & $1.329,35$ & $2.658,70$ & $3.988,05$ & $5.317,40$ \\
\hline B3 & $40.162,78$ & $40.162,78$ & $4.016,28$ & $8.032,56$ & $12.048,83$ & $16.065,11$ \\
\hline B4 & $3.478,08$ & $3.478,08$ & 347,81 & 695,62 & $1.043,42$ & $1.391,23$ \\
\hline $\mathrm{C} 1$ & $32.604,15$ & $41.310,75$ & & & & \\
\hline D1 & & $228.586,15$ & $121.479,63$ & $44.518,71$ & $51.509,80$ & $11.078,01$ \\
\hline D2 & $56.513,86$ & $71.605,30$ & $38.053,86$ & $13.945,62$ & $16.135,60$ & $3.470,22$ \\
\hline D3 & $432.548,39$ & $548.055,95$ & $291.258,39$ & $106.737,63$ & $123.499,40$ & $26.560,53$ \\
\hline D4 & & $121.178,20$ & & $23.600,28$ & $27.306,40$ & $5.872,68$ \\
\hline D5 & & $19.905,01$ & & & & \\
\hline E1 & & $24.698,25$ & $6.174,56$ & $6.174,56$ & $6.174,56$ & $6.174,56$ \\
\hline E2 & & $268.058,34$ & $67.014,59$ & $67.014,59$ & $67.014,59$ & $67.014,59$ \\
\hline E3 & & $19.758,60$ & $4.939,65$ & $4.939,65$ & $4.939,65$ & $4.939,65$ \\
\hline E4 & & $38.419,50$ & $9.604,88$ & $9.604,88$ & $9.604,88$ & $9.604,88$ \\
\hline $\mathrm{F} 1$ & & $13.770,25$ & $7.318,05$ & $2.681,85$ & $3.103,00$ & 667,35 \\
\hline $\mathrm{F} 2$ & $23.630,10$ & $23.630,10$ & $5.907,53$ & $5.907,53$ & $5.907,53$ & $5.907,53$ \\
\hline F3 & & $5.508,10$ & $2.927,22$ & $1.072,74$ & $1.241,20$ & 266,94 \\
\hline G1 & & $27.060,00$ & $6.765,00$ & $6.765,00$ & $6.765,00$ & $6.765,00$ \\
\hline $\mathrm{G} 2$ & $4.347,22$ & $5.508,10$ & $2.927,22$ & $1.072,74$ & $1.241,20$ & 266,94 \\
\hline G3 & $8.694,44$ & $11.016,20$ & $5.854,44$ & $2.145,48$ & $2.482,40$ & 533,88 \\
\hline $\mathrm{H} 1$ & $89.118,01$ & $112.916,05$ & $60.080,01$ & $21.991,17$ & $25.444,60$ & $5.472,27$ \\
\hline TOTAL & $658.276,00$ & $1.665 .661,20$ & $653.124,01$ & $340.095,91$ & $383.340,89$ & $188.949,30$ \\
\hline $\begin{array}{c}\text { Quantidade } \\
\text { Objetos de custo }\end{array}$ & 217.361 & 275.405 & 146.361 & 53.637 & 62.060 & 13.347 \\
\hline $\begin{array}{l}\text { Custo unitário } \\
\text { médio }\end{array}$ & 3,03 & 6,05 & 4,46 & 6,34 & 6,18 & 14,16 \\
\hline
\end{tabular}


A Tabela 4 destaca os valores do custo das principais atividades em ordem decrescente e sua porcentagem. Destacam-se as duas primeiras atividades (D3 e D1), que somadas chegam a mais da metade (53\%) de todo o custo do CME, além da atividade $\mathrm{H} 1$ - Gerenciamento do processo. Essa última atingiu percentual de 7,7\%, sendo a única não diretamente inerente ao processo, entretanto permeia as demais atividades em todos os subprocessos.

Tabela 4: Custo das principais atividades em ordem decrescente. Bauru, SP, Brasil, 2012.

\begin{tabular}{cccc}
\hline Ativ. & Descrição & Valor (R\$) & Total (\%) \\
\hline D3 & Montagem e rotulação dos artigos & $989.689,78$ & $37,40 \%$ \\
D1 & Secagem dos artigos & $411.634,33$ & $15,60 \%$ \\
E2 & Acompanhamento dos ciclos de esterilização & $268.092,61$ & $10,10 \%$ \\
D4 & Recebimento, conferência e montagem roupas & $219.290,97$ & $8,30 \%$ \\
H1 & Gerenciamento do processo & $204.487,31$ & $7,70 \%$ \\
D2 & Conferência dos artigos & $128.134,42$ & $4,80 \%$ \\
C1 & Desinfecção térmica & $72.283,77$ & $2,70 \%$ \\
B3 & Limpeza manual dos artigos & $41.129,10$ & $1,60 \%$ \\
E4 & Verificação da efetividade da esterilização & $38.462,13$ & $1,50 \%$ \\
& Demais atividades & 272208,42 & $10,30 \%$ \\
\hline
\end{tabular}

\section{DISCUSSÃO}

O organograma do hospital em estudo apresenta posições hierárquicas bem definidas, dificultando as tomadas de decisões no nível de supervisão, demonstrando assim forte influência da Teoria Geral da Administração, onde os pressupostos da administração clássica e científica são muito presentes.

A gerência focada em abordagens prescritivas e normativas conduz racionalmente o trabalho, no entanto, mantém o comando dos trabalhadores em uma relação de subordinação e obediência às normas e as prescrições existentes $^{(16)}$.

Um estudo brasileiro evidenciou que as práticas gerenciais desenvolvidas pelos enfermeiros estão concentradas na dimensão técnica da gerência, com ênfase nas atividades de coordenação, supervisão e controle, ressaltando que os preceitos das teorias clássicas da administração ainda norteiam o gerenciamento de enfermagem ${ }^{(17)}$. A superação desse modelo requer estratégias que sejam mais coletivas $e$ participativas envolvendo assim os trabalhadores na compreensão e importância dos custos em seu trabalho.

Dados precisos quanto ao custo das atividades do CME facilitam a identificação dos nós críticos de seu processo e influenciam positivamente nas decisões gerenciais, baseado em informações consistentes, como as disponíveis na Tabela $4^{(12,18)}$.

As informações financeiras intermediárias, de produção, de custos diretos e insumos devem estar disponíveis para a aplicação do custeio $A B C^{(9)}$. Assim sendo, possibilitou-se o ordenamento decrescente dos recursos e evidenciou-se ser o Recurso Pessoal o de maior impacto $(44,5 \%)$, seguido do Material de Consumo e Embalagens (17,8\%) e da Manutenção Equipamentos (12,5\%). Quanto ao Recurso Pessoal, a literatura registra $60 \%{ }^{(5)}$ do custo nas instituições, porcentagem que não se constato no presente estudo. No entanto, destaca-se o impacto nas organizações dos custos com pessoal.

O custo unitário de esterilização de artigos é quase o dobro $(199,7 \%)$ se comparado ao custo unitário de desinfecção de artigos (Tabela 3). Embora economicamente mais viável, não são todos os artigos quem podem passar pelo processo de desinfecção.

O enfermeiro gestor pode considerar uma maior utilização do processo de desinfecção térmica pela viabilidade econômica demonstrada no estudo, sem, no entanto, deixar de seguir as restrições técnicas e científicas inerente ao processamento de artigos. 
O custo da esterilização a vapor saturado sob pressão foi de $\mathrm{R} \$$ 6,05 para cada unidade do artigo médicohospitalar esterilizado, valor relativamente menor que o encontrado em outro trabalho ${ }^{(9)}$, realizado em hospital do interior do Estado de São Paulo.

A Tabela 4 evidencia que o subprocesso Preparo acumula, com apenas duas atividades, mais da metade do valor (53\%) de todo o processo do CME. Nessa etapa ocorre um grande consumo de material para embalagem, entretanto, esse subprocesso é determinado pela absorção de muitas horas dos trabalhadores do CME.

Assim, essa somatória proporciona um grande impacto no custo do processamento dos artigos médicohospitalares, podendo ser reduzida por meio de um sistema informatizado gerando maior agilidade nos registros e identificação dos artigos processados, possibilitando rastreabilidade de materiais por ciclos de esterilização e ainda liberação de etiquetas de identificação das caixas entre outros benefícios ${ }^{(19)}$.

A avaliação da área de Preparo quanto à adequação ergonômica dos mobiliários associada à informatização dos registros pode reduzir o tempo despendido na realização das atividades da área e redução dos custos das $\operatorname{mesmas}^{(17)}$.

Um processo de esterilização seguro e efetivo é essencial no $\mathrm{CME}^{(8)}$. A garantia da segurança do processo bem como sua efetividade evidenciou-se na atividade $\mathrm{H} 1$ - Gerenciamento do processo, correspondendo a 7,7\% do custo das atividades e na atividade E2 Acompanhamento dos ciclos de esterilização indicando a realização desse controle e consumindo pouco mais de $10 \%$ dos custos das atividades (Tabela 4). 0 gerenciamento de todo o processamento de materiais é de responsabilidade do Enfermeiro e visa garantir uma assistência eficiente e de qualidade ${ }^{(6,20)}$.

Nesse sentido, destaca-se que o processo de esterilização é mais seguro, pois possui validação das autoclaves e indicadores térmicos, químicos e biológicos da eficácia da esterilização ${ }^{(5)}$. Essa configuração proporciona segurança ao processo, entretanto, aumento do custo unitário dos artigos. Há que se considerar, no entanto, que o custo final em função da segurança se justifica.

Os percentuais encontrados para as duas atividades acima citadas sinalizam que existe um esforço consciente para a garantia da qualidade no processamento de materiais no CME. Este achado ratifica a informação demonstrada no diagnóstico institucional, indicando o alinhamento da unidade à estratégia institucional na gestão por processos.

\section{CONCLUSÕES}

O custeio $A B C$ é aplicável ao CME e possibilita o surgimento de evidências que balizam a tomada de decisão no contexto da prática gerencial, garantindo a realização de análise do processo produtivo por meio do custeio das atividades, e determinando intervenções decisivas e com impacto na gestão de custos.

$O$ custeio $A B C$ no $C M E$ prescinde de um sistema de informação mínimo de custos a fim de viabilizar sua aplicação e ser uma primeira aproximação do enfermeiro gestor aos relatórios de custo. Ademais, agregou-se muitos dados e informações provenientes de diferentes departamentos da instituição hospitalar para composição do custeio $A B C$, que pode ser um fator que dificulte a sua aplicação nas instituições hospitalares.

A prática gerencial no CME deve se valer do custeio $A B C$ como fonte de evidências na tomada de decisão para investimentos em estrutura física, recursos humanos, tecnológicos e reengenharia de processos.

$O$ custeio $A B C$ poderia ainda contribuir com os relatórios financeiros das unidades hospitalares na elaboração de estratégias de controle e uso racional dos artigos por meio da identificação do custo do estoque de artigos nessas unidades; servir de argumentação para o investimento em tecnologia da informação, na aquisição de equipamentos mais modernos e ergonômicos que podem contribuir de maneira significativa para redução dos custos das atividades da área de preparo da unidade, agilizando registro das identificações, reduzindo o tempo 
de secagem dos artigos médico-hospitalares e otimizando a quantidade de horas utilizadas pelos trabalhadores no processamento desses artigos.

A avaliação do valor agregado das atividades possibilitaria outras análises e evidências, entretanto não houve condições de implementá-la, indicando lacunas para estudos futuros.

\section{REFERÊNCIAS}

1. Zuliani LL, Jericó MC, Castro LC, Soler ZASG. Consumo e custo de recursos materiais em unidades pediátricas de terapia intensiva e semi-intensiva. Rev. bras. enferm. [Internet]. 2012 [acesso em: 30 jun 2015];65(6):969-76. Disponível em: http://dx.doi.org/10.1590/50034-71672012000600013. 2. Bonacim CAG, Araujo AMP. Gestão de custos aplicada a hospitais universitários públicos: a experiência do Hospital das Clínicas da Faculdade de Medicina de Ribeirão Preto da USP. Rev. Adm. Pública [Internet]. 2010 [acesso em: 30 jun 2015];44(4):903-31. Disponível em: http://dx.doi.org/10.1590/S0034-76122010000400007. 3. Castilho V, Fugulin FMT, Gaidzinski RR. Gerenciamento de custos nos serviços de Enfermagem. In: Kurcgant $P$, coordenador. Gerenciamento em Enfermagem. $2^{\underline{a}}$ ed. Rio de Janeiro: Guanabara Koogan; 2011. p. 169-71.

4. Oliveira WT, Rodrigues AVD, Haddad MCL, Vannuch MTO, Taldivo MA. Conceptions of nurses from a public university hospital regarding the cost management report. Rev. esc. enferm. USP [Internet] 2012 [acesso em: 30 jun 2015];46(5):1184-91. Disponível em: http://dx.doi.org/10.1590/S0080-62342012000500021. 5. Sociedade Brasileira de Enfermeiras de Centro Cirúrgico, Recuperação Anestésica e Centro de Material e Esterilização. Práticas recomendadas. 6ạ ed. São Paulo: SOBECC; 2013. 6. Possari JF. Centro de Material e Esterilização: Planejamento, Organização e Gestão. 4ạ ed. São Paulo: látria; 2010.

7. Ching HI. Manual de custos de instituições de saúde: sistemas tradicionais de custos e sistema de custeio baseado em atividades (ABC). 2ª ed. São Paulo: Atlas; 2010.

8. Costa JA, Fugulin FMT. Atividades de enfermagem em centro de material e esterilização: contribuição para o dimensionamento de pessoal. Acta paul. enferm. [Internet]. 2011 [acesso em: 30 jun 2015];24(2):249-56. Disponível em: http://dx.doi.org/10.1590/S0103-21002011000200015.

9. Jericó MC, Castilho V. Gerenciamento de custos: aplicação do método de Custeio Baseado em Atividades em Centro de Material Esterilizado. Rev. esc. enferm. USP [Internet]. 2010 [acesso em: 30 jun 2015];44(3):745-52. Disponível em: http://dx.doi.org/10.1590/S0080-62342010000300028.
Concluindo, o custeio $A B C$ proporcionou a possibilidade de vislumbrar o possível investimento proposto de forma temporal (pequeno, médio ou longo prazo), identificar pontos críticos no processo de trabalho com vistas a reengenharia e pelas proposições aqui apresentadas, verifica-se ser o custeio $A B C$ uma ferramenta eficiente para a prática gerencial baseada em evidências em um CME.

10. Miranda GJ, Carvalho CE, Martins VF, Faria AF. Custeio ABC no ambiente hospitalar: um estudo nos hospitais universitários e de ensino brasileiros. Rev. contab. finanç. [Internet]. 2007 [acesso em: 30 jun 2015];18(44):33-43. Disponível em: http://dx.doi.org/10.1590/S1519-70772007000200004.

11. Hada MS, Chakravarty A, Mukherjee P. Activity based costing of diagnostic procedures at a nuclear medicine center of a tertiary care hospital. Indian J Nucl Med [Internet]. 2014 [acesso em: 30 jun 2015];29(4):241-5. Disponível em: http://dx.doi.org/10.4103/0972-3919.142627.

12. Yereli NA. Activity-based costing and its application in a Turkish university hospital. AORN J [Internet]. 2009 [acesso em: 30 jun 2015];89(3):573-91. Disponível em:

http://dx.doi.org/10.1016/j.aorn.2008.09.002.

13. Cannavacciuolo L, Illario M, Ippolito A, Ponsiglione C. An activity-based costing approach for detecting inefficiencies of healthcare processesnull. Bus Process Manag J [Internet]. 2015 [acesso em: 30 jun 2015];21(1):55-79. Disponível em: http://dx.doi.org/10.1108/BPMJ-11-2013-0144.

14. Yin RK. Estudo de caso: planejamento e métodos. 4a ed. Porto Alegre: Bookman; 2010.

15. Polit DF, Beck CT. Fundamentos de pesquisa em Enfermagem. Avaliação de evidências para a prática de enfermagem. 7ạ ed. Porto Alegre: Artmed; 2011.

16. Matos E, Pires D. Teorias administrativas e organização do trabalho: de Taylor aos dias atuais, influências no setor saúde e na enfermagem. Texto contexto - enferm. [Internet]. 2006 [acesso em: 30 jun 2015];15(3):508-14. Disponível em: http://dx.doi.org/10.1590/S0104-07072006000300017. 17. Santos JLG, Garlet ER, Lima MADS. Revisão sistemática sobre a dimensão gerencial no trabalho do enfermeiro no âmbito hospitalar. Rev. Gaúcha Enferm. [Internet]. 2009 [acesso em: 30 jun 2015];30(3):525-32. Disponível em: http://www.seer.ufrgs.br/RevistaGauchadeEnfermagem/articl e/view/7879.

18. Oller GASAO, Baldo LEK, Chaves LD, Parro MC, Jenal S, Chaves LDP. Enfermagem e custos: uma revisão da literatura nacional. CuidArte, Enferm. 2010;4(1):36-43.

19. Sancinetti TR, Gatto MAF. Parâmetros de produtividade de um centro de material e esterilização. Rev. esc. enferm. USP 
[Internet]. 2007 [acesso em: 30 jun 2015];41(2):264-70.

http://dx.doi.org/10.1590/S0080-62342007000200013.

20. Albano TC, Freitas JB. Participação efetiva do enfermeiro no planejamento: foco nos custos. Rev. bras. enferm. [Internet]. 2013 [acesso em: 30 jun 2015];66(3):372-77. Disponível em: http://dx.doi.org/10.1590/\$0034-71672013000300011.

Recebido: 02/12/2013.

Aceito: 04/03/2015.

Publicado: 30/06/2015. 